\title{
In vitro hemocompatibility evaluation of the HeartWare ventricular assist device under systemic, pediatric and pulmonary support \\ conditions
}

${ }^{1,2,3}$ Chris H. H. Chan, ${ }^{1,3}$ Katrina K. Ki, ${ }^{1,3}$ Ian Y. Chu, ${ }^{1,3}$ Joshua Rolls, ${ }^{1,2}$ Sasha Morris, ${ }^{1,3}$ Talvin J. Lee, ${ }^{1}$ Stefan Bindorfer, ${ }^{1,2}$ Jo P. Pauls, ${ }^{4,5}$ Iki Idachi, ${ }^{1,3}$ John F. Fraser

${ }^{1}$ Critical Care Research Group, The Prince Charles Hospital, Australia

${ }^{2}$ School of Engineering and Built Environment, Griffith University, Australia

${ }^{3}$ Faculty of Medicine, The University of Queensland, Australia

${ }^{4}$ Division of Congenital Heart Surgery, Texas Children's Hospital, USA

${ }^{5}$ Michael E. DeBakey Department of Surgery, Baylor College of Medicine, USA

Authors' contributions

- Chris H.H. Chan: Analysis/data interpretation, drafting/critical revision/approval of article

- Katrina K. Ki: Data interpretation, drafting/critical revision/approval of article

- Ian Y. Chu: Analysis/data interpretation, drafting/critical revision/approval of article

- Joshua Rolls: data collection, approval of article

- Sasha Morris: data collection, approval of article

- Talvin J. Lee: data collection and interpretation, approval of article

- Stefan Bindorfer: data collection, approval of article

- Jo P. Pauls: Approval of article

- Iki Idachi: Approval of article

- John F. Fraser: Approval of article

Running Headline: Hemocompatibility of HVAD in various supports

Corresponding author:

Dr. Chris Chan Hoi Houng 


\section{Griffith University}

Critical Care Research Group

Clinical Sciences Building, Level 3

The Prince Charles Hospital

Chermside, QLD 4032

Email: chris houng@hotmail.com

Tel: +61 424887256

Conflicts of Interest and Source of Funding: none declared. 


\section{In vitro hemocompatibility evaluation of the HeartWare ventricular assist device under systemic, pediatric and pulmonary support conditions}

The development of adult use right ventricular assist devices (RVADs) and pediatric left ventricular assist devices (pediatric LVADs) have significantly lagged behind compare to adult use left ventricular assist devices (LVADs). The HeartWare Ventricular Assist Device (HVAD) intended to be used for adult's systemic support, is increasingly used off-label for adult pulmonary and pediatric systemic support. Due to different hemodynamics and physiology, however, the HVAD's hemocompatibility profiles can be drastically different when used in adult pulmonary circulation or in children, compared to its intended usage state, which could have a direct clinical and developmental relevance. Taking these considerations in mind, we sought to conduct in vitro hemocompatibility testing of HVAD in adult systemic, pediatric systemic and adult pulmonary support conditions. Two HVADs coupled to custom-built blood circulation loops were tested for 6 hours using bovine blood at $37^{\circ} \mathrm{C}$ under adult systemic, pediatric systemic, and adult pulmonary flow conditions (flow rate $=5.0,2.5$ and 4.5 L/min; differential pressure $=100,69$, and $20 \mathrm{mmHg}$, respectively). Normalized index of hemolysis for adult systemic, pediatric systemic and adult pulmonary conditions were $0.0083,0.0039$ and $0.0017 \mathrm{~g} / 100 \mathrm{~L}$, respectively. No significant difference was seen in platelet activation for these given conditions. High molecular weight von Willebrand factor multimer degradation was evident in all conditions $(p<0.05)$. In conclusion, alterations in the usage mode produce substantial differences in hemocompatibility of the HVAD. These findings would not only have clinical relevance but will also facilitate future adult use RVAD and pediatric LVAD development. 
Key Words: Ventricular assist device, systemic, pediatric, pulmonary, hemolysis, platelet activation, HMW vWF degradation

\section{Introduction}

Over the last few decades, left ventricular assist devices (LVADs) have become a standard treatment option for end-stage systolic heart failure in adults, either as a bridge-to-transplant or destination therapy. ${ }^{1}$ However, the field of mechanical circulatory support is lagging behind in the development of adult use right ventricular assist devices (RVADs) and pediatric left ventricular assist device (pediatric LVADs). ${ }^{2,3}$ This frustrating reality is reflected in the fact that there is only short-term option Impella RP (Abiomed, Danvers, USA) for adult use right ventricular support and Berlin Heart EXCOR (Berlin Heart, Berlin, Germany) for pediatric support were approved by U.S. Food and Drug Administration (FDA). History has shown that the development of RVAD and pediatric LVAD is far more challenging than that of adult LVADs for multifactorial reasons. ${ }^{3,4}$

Due to the lack of a clinically available long-term implantable rotary RVAD and pediatric LVAD, HeartWare Ventricular Assist Device (HVAD) (Medtronic, Minneapolis, USA) has been used as an RVAD or pediatric LVAD in an off-label fashion. ${ }^{5-8}$ Owing to its compact design, HVAD has been increasingly used as a bridge to transplantation in children and adolescents with end-stage heart failure and as an RVAD support by reducing the pump speed to accommodate lower resistance in the adult pulmonary circulation. ${ }^{9-12}$ In pediatric patients with a low body surface area $(B S A)<1.0 \mathrm{~m}^{2}$, typically range between $0.6-0.9 \mathrm{~m}^{2}$ and cardiac output required between $2.1-3.0 \mathrm{~L} / \mathrm{min}$, pump speeds of the HVAD were adjusted to between 2300 - 2450 RPM. ${ }^{13-15}$ In addition, previous in vitro studies for pediatric HVAD usage employed $2.5 \mathrm{~L} / \mathrm{min}$ as a typical average flow for pediatric systemic condition. ${ }^{6,16}$ In 
biventricular failure patients, low pump speed of the HVAD as RVAD is used typically 1900 RPM in order to produce low pressure output between 14 - $23 \mathrm{mmHg}$ and normal cardiac output between 4 - 5 L/min. 9,10,17

While appropriate adjustments to HVAD's operating specifications have reported success supporting right heart failure and pediatric patients, it is potentially associated with a high rates of major adverse events; multisystem organ failure, neurological dysfunction, respiratory failure, pump thrombosis, infection, stroke, and major bleeding. ${ }^{10,18-22}$ This study was designed to evaluate the effect of HVAD on hemolysis, platelet activation and vWF degradation in adult systemic, pediatric systemic and adult pulmonary flow conditions. Following the standard practice for assessing blood damage in continuous-flow pumps per ASTM F1841-97, this study aimed to provide benchmark values for future RVAD and pediatric LVAD development. ${ }^{23,24}$

\section{Materials and Methods}

Two blood circulatory loops (BCLs) were built and illustrated in Figure 1. The study was conducted in compliance with the ASTM standards for blood selection (ASTM F1830-97) and in vitro blood pump evaluation (ASTM F1841-97). ${ }^{23,24}$ Four repeat tests were conducted for adult systemic, pediatric systemic and adult pulmonary support conditions.

\section{Preparation of test blood}

Bovine blood was obtained from live cows (Serum Australis Pty Ltd, Inc., NSW, Australia) via venipuncture and collected into an anticoagulated blood bag containing $14 \%$ citrate phosphate dextrose adenine (SSS Australia Health Supplies, Australia). Blood sample hematocrit levels were adjusted to $30 \pm 2 \%$ using phosphate-buffered saline (Sigma-Aldrich, St. Louis, MO, USA) as necessary to reduce variation between 
samples and antibiotic solution (50 mg/L gentamycin, Sigma-Aldrich, St. Louis, MO, USA) was added to prevent bacterial contamination. ${ }^{25}$ The total time elapsed between blood collection and initiating test procedures was less than 24 hours.

\section{Blood circulation loops}

Two explanted HVADs were utilized throughout the study and assigned randomly to the different operating conditions. The in vitro BCLs (total length of $1200 \mathrm{~mm}$ ) comprised of non Di 2-ethylhexyl phthalate (non-DEHP) E-3603 Tygon tubing ( $\varnothing 9.5$ $\mathrm{mm}$ and $\varnothing 12.7 \mathrm{~mm})($ ACF00028 and ACF00036, Gallay medical and scientific, Australia) connected to a custom-made blood reservoir (Figure 1). The volume of blood in each loop was $450 \pm 50 \mathrm{~mL}$. An ultrasonic flow meter (ME9PXL1153; Transonic Systems Inc., NY, USA) was used to monitor pump flow, while inlet and outlet pressures were monitored with piezoresistive pressure transducers (PX181B015C5V; Omega Engineering, Stamford, CT, USA). After each experiment, the BCLs were washed with saline and rinsed in $0.6 \%$ Medizyme solution (Whiteley Medical Pty Ltd, NSW, Australia) for an hour to dissolve all possible blood residue. The BCLs were washed with deionized water four times and rinsed with saline before blood infusion through a $40 \mu \mathrm{m}$ blood transfusion filter (SQ40, Haemonetics, MA, USA) to prevent microaggregates, clots, and contaminated debris such as animal fur and skin tissue gathered during blood collection. Apart from the HVADs, the BCLs were immersed in a water bath following blood infusion and maintained at $37^{\circ} \mathrm{C}$. Blood was circulated for 6 hours. Remaining bovine blood was kept in a blood bag and warmed in the water bath as a static control.

Hemodynamics and data acquisition 
Due to lower cardiac output in pediatric systemic circulation and lower pressure output of adult pulmonary circulation are needed, clinicians turn down the pump speeds of the HVAD to meet these flow conditions. Pump speeds are reported average values of approximately average $2400 \mathrm{rpm}$ for pediatric systemic support and $1900 \mathrm{rpm}$ for adult pulmonary support. 6,9,10,26 Pressure and flow curves for blood flow generated by the pump at various speed throughout a wide range of pressures are shown in Figure 2. Subsequently, the identified pump speed for adult systemic, pediatric systemic and adult pulmonary HVAD conditions were used at 3200, 2400, 1900 RPM, respectively. The hemodynamic parameters for adult systemic, pediatric systemic and adult pulmonary flow regimes of the HVAD were controlled such that: the pump flow was set at 5, 2.5 and $4.5 \mathrm{~L} / \mathrm{min}$ respectively for all experiments; the differential pressure across the pump, $\Delta P$, was adjusted to 100,69 and $20 \mathrm{mmHg}$ using a resistance clamp (Table 1). Blood samples were taken at 5 min after the pumps turned on and later at hourly intervals for $6 \mathrm{~h}$ via a sampling port in the blood reservoir. The first milliliter was discarded and then collected a second draw of $5 \mathrm{~mL}$, which was used for the assays as described.

Hemolysis assay

The Harboe assay was used for determining hemolysis. ${ }^{27}$ The methods were explained in detail previously. ${ }^{28}$ Plasma free hemoglobin $(p f H b)$ was measured with a UV/visible spectrophotometer (SmartSpec plus, Bio-Rad, Hercules, CA, USA) at 380, 415 and $450 \mathrm{~nm}$ wavelengths. Results were averaged and expressed as mean \pm standard error of mean (SEM). The $p f H b$ and normalised index of hemolysis $(N I H)$ was calculated as described by equations (1) and (2), respectively, where $V$ is the blood loop volume, $\mathrm{Q}$ is the flow rate, $\mathrm{Ht}$ is the hematocrit and $\mathrm{T}$ is the sampling time. 


$$
\begin{gathered}
\text { pfHb }\left(\frac{m g}{d L}\right)=(167.2 \times A 415-83.6 \times A 380-83.6 \times A 450) \times\left(\frac{1}{10}\right) \times\left(\frac{1}{\text { dilution in } 0.1 \% N a 2 C O 3}\right) \\
N I H=\Delta p f H b \times V \times \frac{100-H t}{100} \times \frac{100}{Q \times \Delta T}(2)
\end{gathered}
$$

Platelet activation assay

A $100 \mu \mathrm{L}$ aliquot of the every $120 \mathrm{~min}$ blood samples were fixed with Streck solution (Abacus DX, Australia) at 1:1 volume ratio to preserve cellular antigen expression of platelets. In parallel, $1 \mathrm{~mL}$ blood was treated with $5 \mu \mathrm{M}$ phorbol 12-myristate 13acetate (PMA) (Sigma-Aldrich, St. Louis, MO, USA) for 60 min at $37^{\circ} \mathrm{C}$ serving as a positive control. The $20 \mu \mathrm{L}$ fixed blood samples were then stained with $5 \mu \mathrm{L}$ of 1:50 diluted CAPP2A antibody $(1.0 \mathrm{mg} / \mathrm{dL}$, an anti-ruminant CD41/61 antibody; In Vitro Technologies Pty. Ltd, Australia), as staining and analyzing methods were explained in detail previously. ${ }^{28,29}$ Samples were acquired and analyzed using a four laser BD Fortessa X-20 Flow Cytometry Cell Analyzer (BD Bioscience, Franklin Lakes, NJ, USA). Data were analyzed with FACSDiva 6.1.3 software.

Agarose Gel Electrophoresis and Immunoblotting for high molecular weight vWF multimers

Plasma was prepared by centrifuging every $120 \mathrm{~min}$ blood samples for $7 \mathrm{~min}$ at 4200 $\times \mathrm{g}$ at room temperature. Platelet-poor plasma (PPP) was prepared by further centrifuging plasma for $5 \mathrm{~min}$ at $15,000 \times \mathrm{g}$ to remove debris. A mixture of $10 \mu \mathrm{L}$ PPP and $30 \mu \mathrm{L}$ sample buffer was subjected to electrophoresis, protein blotting transfer, VWF specific staining per methods explained in detail previously. ${ }^{28,29}$ The VWF multimers were visualized with the ChemiDoc XRS scanner (Bio-Rad Laboratories, CA, US). The degradation of HMW vWF multimers was quantified by densitometric analysis using ImageJ software (version 1.47, NIH, Bethesda, MD, US) and 
degradation in \% HMW vWF multimer density was obtained by comparing results from each time point against the 5 min (baseline) and expressed as mean percentage change \pm SEM

\section{Statistical Analysis}

GraphPad Prism 7.0 (GraphPad Software, Santiago, US) was used for all statistical and graphic representation. A two-way repeated measures analysis of variance (RMANOVA) was conducted to compare the changes in hemolysis, platelet activation and VWF degradation amongst the three conditions over the 6-hour duration. All data were normalized and compared to values obtained at $5 \mathrm{~min}$ as a baseline. Following detection of statistical significance, a Tukey's follow-up multiple comparisons was conducted. The NIH was found to have a normal distribution and was analyzed by one-way ANOVA with a Tukey's follow-up multiple comparisons. Statistical significance was determined at $p<0.05$.

\section{Results}

Hemolysis

Hemolysis increased significantly over time for all three conditions $(p<0.05)$ except for the static control (Figure $3 \mathrm{~A}$ ). $\Delta p f \mathrm{Hb}$ in the adult systemic condition was significantly increased from $180 \mathrm{~min}$ to $360 \mathrm{~min}$ compared to its baseline $(p<0.05) . \Delta p f H b$ in the pediatric systemic and adult pulmonary conditions were significantly increased at 360 min only compared to its baseline $(p<0.05)$. The $N I H$ for adult systemic condition = $0.0083 \pm 0.0032 \mathrm{~g} / 100 \mathrm{~L}$, pediatric systemic condition $=0.0039 \pm 0.0016 \mathrm{~g} / 100 \mathrm{~L}$, adult pulmonary condition $=0.0017 \pm 0.0007 \mathrm{~g} / 100 \mathrm{~L}($ Figure 3B $)$.

Platelet Activation 
The level of platelet activation detected by CAPP2A antibody remained the same over time for all three flow conditions $(n=4)$ and static condition $(n=6)$ (Figure 4). For the PMA-stimulated positive control, $24.7 \pm 17.6 \%(n=6)$ of activated platelets detected by CAPP2A antibody.

High molecular weight vWF degradation

A significant degradation of HMW vWF multimers (within red dotted box) were found over time in those given conditions $(p<0.05)$ (Figure 5A). In the pediatric systemic and adult systemic conditions, HMW vWF density was significantly degraded at 360 min only compared to $5 \mathrm{~min}(\mathrm{p}<0.05)$. The adult pulmonary condition also had significant degraded from 5 min at $240 \mathrm{~min}$ and $360 \mathrm{~min}(p<0.05)$. No significant differences in HMW vWF degradation were found in the static control over time (Figure 5B).

\section{Discussion}

Options for durable mechanical circulatory support for patients with chronic right ventricular failure or pediatric patients are limited. Currently, there are no clinically available long-term implantation RVAD or pediatric LVAD, and Impella RP for pulmonary support and Berlin Heart EXCOR for pediatric support are FDA-approved for short-term hospital inpatient support. 2,3 Thus, the usage of HVAD as an RVAD and pediatric LVAD substitute have become common practice..$^{9,10,30}$

There is an urgent need for the development of long-term, implantable RVADs and pediatric LVADs that are specifically designed for the unique characteristic of the adult pulmonary and pediatric systemic circulation. When developing a new VAD, 
hemodynamic-induced blood damage is one for the most critical elements for assessment to determine the device's hemocompatibility. ${ }^{31}$ However, there are no benchmark values available from baseline control device to assess hemocompatibility and guide the development of new RVAD and pediatric LVAD to date. Therefore, this study aimed to help VAD developers to recognize the potential need for design iterations without delay which serves as a benchmark in the early stage of the development process.

The present study aimed to evaluate the blood damage caused by the HVAD under these off-label pediatric systemic and adult pulmonary conditions, as well as its designated adult systemic condition. Given the fact that the HVAD has clinically been used as an RVAD and for children, the results of this study, which strictly follows the ASTM standard for in vitro continuous flow blood pump evaluations, will serve as a hemocompatible performance benchmark for upcoming pediatric and pulmonary specific devices. ${ }^{23,24}$

To provide in vitro benchmark data for adult and pediatric LVAD development, we previously tested the CentriMag blood pump (St. Jude Medical Inc., St. Paul, MN) for standard adult systemic flow conditions ${ }^{32}$, the EXCOR VAD (Berlin Heart Inc., The Woodland, TX), and HeartMate II (St. Jude Medical Inc.) for pediatric systemic flow conditions. ${ }^{16}$ In addition, we also previously tested the HVAD as RVAD support by reducing the right pump speed or restricting the diameter of the outflow graft by "banding" using human blood. ${ }^{26}$ However, the HVAD has not been previously evaluated for adult systemic, pediatric systemic and adult pulmonary flow conditions under the same experimental set-up. 
Shear stresses generated by the rotating impeller of the HVAD has many implications for blood damage. ${ }^{29}$ Our in vitro results have shown that all conditions were below recommended hemolysis threshold $(\mathrm{NIH}<0.01 \mathrm{~g} / 100 \mathrm{~L})$ and the adult systemic condition produced a significantly higher $\mathrm{NIH}$ compared to the pediatric systemic and adult pulmonary conditions. ${ }^{31}$ With the highest impeller speed among three given conditions, the adult systemic condition is expected to generate the most mechanical shear stress. ${ }^{33}$ In previous study, a new version Infant Jarvik 2015 (Jarvik Heart, Inc., New York, NY), the only implantable pediatric LVAD specifically designed for kids, has substantially reduced hemolysis compared to the first version design infant Jarvik 2000 VAD when the impeller speed reduced from 28,000 RPM to below 20,000 RPM. ${ }^{4}$ The $\mathrm{NIH}$ of the adult pulmonary condition reflected the results of a previous experiment under similar conditions $(0.0033 \pm 0.00026 \mathrm{~g} / 100 \mathrm{~L}$ at $1920 \mathrm{RPM}){ }^{26}$ While the adult systemic condition produced results comparable with a previous study $(0.0082 \pm$ $0.0045 \mathrm{~g} / 100 \mathrm{~L}$ at $2800 \mathrm{RPM})$, however, the same study demonstrated a much higher pediatric NIH despite having lower rotational speed $(0.0175 \pm 0.0097 \mathrm{~g} / 100 \mathrm{~L}$ at 2200 RPM). ${ }^{6}$ The discrepancy may be the result of longer duration time of experiments (6 vs 46 hours).

This study found no significant platelet activation in any of the flow conditions. Pump thrombosis is a major complication that is common in all HVAD implantations, particularly in pediatric systemic and adult pulmonary patients where the low flow state potentially increases stagnant regions of blood within the pump. ${ }^{19,34,35}$ However, bovine platelets have been shown to be less susceptible to shear stress than human platelets and a shear rate threshold must be reached to activate platelets. ${ }^{36}$ It is likely that the experiments conducted did not produce enough shear stress for bovine platelet activation, especially given the short run times of 6 hours. ${ }^{29}$ Compared with 
clinical HVAD use where patients are used from weeks to years, the lack of platelet activation in this study might not reflect the clinical reality.

Blood samples of all conditions caused a similar trend of HMW vWF multimer degradation of circulating blood throughout $360 \mathrm{~min}$ in vitro testing. This phenomenon may suggest that the HMW vWF bands were cleaved into smaller fragments. ${ }^{37,38}$ In adult pulmonary circulation, low pump speed might leads to hydrodynamic instability of the HVAD rotor which potentially caused more HMW VWF degradation when compared to other two conditions. Our results seem to be consistent with clinical observations that all HVAD recipients develop acquired von Willebrand syndrome, demonstrated by the HMW vWF multimer degradation during in vitro testing. ${ }^{39,40}$

Major limitations of the present study primarily stem from the fact that the in vitro test conditions used is not a perfect representation of a real hemodynamic condition in pediatric patients and right ventricular failure patients. Given the lack of in vitro benchmark data for children and right ventricular support, nonetheless, we believe that this study will serve helpful information necessary for RVAD and pediatric LVAD development, which significantly lags behind that for adults. In addition, the HVADs used in this study were explanted devices from patients. Therefore, HVADs were randomized between the three conditions to eliminate any risk of the individual pump contributing to the results.

\section{Conclusion}

We have conducted hemocompatibility testing on HVAD as LVAD, pediatric LVAD and RVAD by mimicking the commonly used adult systemic, pediatric systemic and adult pulmonary flow conditions clinically. Our in vitro model using bovine blood under 
ASTM standard testing conditions demonstrated a higher hemolysis profile in HVAD's

FDA approved used in adult systemic condition, in comparison to the adult pulmonary and pediatric systemic conditions. While no significant platelet activation was observed over time in any condition, significant degradation of HMW vWF under all conditions was evident. These results can serve as hemocompatible performance benchmarks for future development of LVADs, pediatric LVADs and RVADs.

\section{Funding}

The authors would like to recognise the financial assistance provided by the ACTIONS National Health and Medical Research Council Centre for Research Excellence (APP1079421), The Prince Charles Hospital Foundation Team Grant (TM2017-04) and New Investigator Grant (NI2019-57). Chris H.H. Chan is supported by The Prince Charles Hospital Foundation Research Fellowship (RF2019-02).

\section{Reference}

1. Rogers JG, Pagani FD, Tatooles AJ, et al: Intrapericardial Left Ventricular Assist Device for Advanced Heart Failure. The New England journal of medicine 376: 451-460, 2017.

2. Burki S, Adachi I: Pediatric ventricular assist devices: current challenges and future prospects. Vascular health and risk management 13: 177-185, 2017.

3. Karimov JH, Sunagawa G, Horvath D, Fukamachi K, Starling RC, Moazami N: Limitations to Chronic Right Ventricular Assist Device Support. The Annals of thoracic surgery 102: 651-8, 2016.

4. Baldwin JT, Adachi I, Teal J, et al: Closing in on the PumpKIN Trial of the Jarvik 2015 Ventricular Assist Device. Seminars in thoracic and cardiovascular surgery Pediatric cardiac surgery annual 20: 9-15, 2017.

5. Silva Enciso J, Tran HA, Brambatti M, Braun OO, Pretorius V, Adler ED: Management of RVAD Thrombosis in Biventricular HVAD Supported Patients: Case Series. ASAIO journal (American Society for Artificial Internal Organs : 1992) 65: e36-e41, 2019.

6. Granegger M, Thamsen B, Schloglhofer T, et al: Blood trauma potential of the HeartWare Ventricular Assist Device in pediatric patients. The Journal of thoracic and cardiovascular surgery, 2019.

7. Krabatsch T, Potapov E, Stepanenko A, et al: Biventricular circulatory support with two miniaturized implantable assist devices. Circulation 124: S179-86, 2011. 
8. Pac M, Kocabeyoglu SS, Kervan U, et al: Third Generation Ventricular Assist Device: Mid-Term Outcomes of the HeartWare HVAD in Pediatric Patients. Artificial organs 42: 141-147, 2018.

9. Strueber M, Meyer AL, Malehsa D, Haverich A: Successful use of the HeartWare HVAD rotary blood pump for biventricular support. The Journal of thoracic and cardiovascular surgery 140: 936-7, 2010.

10. Tran HA, Pollema TL, Silva Enciso J, et al: Durable Biventricular Support Using Right Atrial Placement of the HeartWare HVAD. ASAIO journal 64: 323-327, 2018.

11. Saito S, Sakaguchi T, Miyagawa S, et al: Biventricular support using implantable continuous-flow ventricular assist devices. The Journal of heart and lung transplantation : the official publication of the International Society for Heart Transplantation 30: 475-8, 2011.

12. Miera O, Potapov EV, Redlin M, et al: First experiences with the HeartWare ventricular assist system in children. The Annals of thoracic surgery 91: 1256-60, 2011.

13. Miera $\mathrm{O}$, Kirk $\mathrm{R}$, Buchholz $\mathrm{H}$, et al: A multicenter study of the HeartWare ventricular assist device in small children. The Journal of heart and lung transplantation : the official publication of the International Society for Heart Transplantation 35: 679-81, 2016.

14. Adachi I, Zea-Vera $\mathrm{R}$, Tunuguntla $\mathrm{H}$, et al: Centrifugal-flow ventricular assist device support in children: A single-center experience. The Journal of thoracic and cardiovascular surgery 157: 1609-1617.e2, 2019.

15. Adachi I, Burki S, Horne D, et al: The miniaturized pediatric continuous-flow device: Preclinical assessment in the chronic sheep model. The Journal of thoracic and cardiovascular surgery 154: 291-300, 2017.

16. Chan $\mathrm{CHH}$, Diab S, Moody K, et al: In Vitro Hemocompatibility Evaluation of Ventricular Assist Devices in Pediatric Flow Conditions: A Benchmark Study.

Artificial organs, 2018.

17. Maynes EJ, O'Malley TJ, Patel P, et al: Right Atrial versus Right Ventricular HeartWare HVAD Position in Patients on Biventricular HeartWare HVAD Support: A Systematic Review. Artificial organs, 2020.

18. Rosenthal DN: Erratum to "Adverse Events in Children Implanted with Ventricular Assist Devices in the US: Data from the Pediatric Interagency Registry for Mechanical Circulatory Support (PEDIMACS)". The Journal of heart and lung transplantation 36: 116, 2017.

19. Aissaoui N, Borgermann J, Gummert J, Morshuis M: HeartWare continuousflow ventricular assist device thrombosis: the Bad Oeynhausen experience. The Journal of thoracic and cardiovascular surgery 143: e37-9, 2012.

20. Susen S, Rauch A, Van Belle E, Vincentelli A, Lenting PJ: Circulatory support devices: fundamental aspects and clinical management of bleeding and thrombosis. Journal of thrombosis and haemostasis: JTH 13: 1757-67, 2015.

21. Kirklin JK, Pagani FD, Kormos RL, et al: Eighth annual INTERMACS report: Special focus on framing the impact of adverse events. The Journal of heart and lung transplantation 36: 1080-1086, 2017.

22. Shah $\mathrm{P}, \mathrm{Ha} \mathrm{R}$, Singh $\mathrm{R}$, et al: Multicenter experience with durable biventricular assist devices. The Journal of heart and lung transplantation 37: 1093$1101,2018$.

23. ASTM. F1841 - 97 (2017): Standard Practice for Assessment of Hemolysis in Continuous Flow Blood Pumps. 
24. ASTM. F1830 - 97 (2017): Standard Practice for Selection of Blood for in vitro Evaluation of Blood Pumps.

25. Tayama E, Shimono T, Makinouchi K, et al: Reconsideration of total erythrocyte destruction phenomenon. Artificial organs 21: 704-9, 1997.

26. Chan $\mathrm{CHH}$, Nandakumar D, Balletti N, et al: In Vitro Hemocompatibility Evaluation of Modified Rotary Left to Right Ventricular Assist Devices in Pulmonary Flow Conditions. ASAIO journal, 2019.

27. Han V, Serrano K, Devine DV: A comparative study of common techniques used to measure haemolysis in stored red cell concentrates. Vox sanguinis 98: 11623, 2010.

28. Chan $\mathrm{CHH}$, Diab S, Moody K, et al: In Vitro Hemocompatibility Evaluation of Ventricular Assist Devices in Pediatric Flow Conditions: A Benchmark Study. Artificial organs 42: 1028-1034, 2018.

29. Chan $\mathrm{CHH}$, Pieper IL, Robinson CR, Friedmann Y, Kanamarlapudi V, Thornton CA: Shear Stress-Induced Total Blood Trauma in Multiple Species. Artificial organs 41: 934-947, 2017.

30. Conway J, Miera O, Adachi I, et al: Worldwide Experience of a Durable Centrifugal Flow Pump in Pediatric Patients. Seminars in thoracic and cardiovascular surgery 30: 327-335, 2018.

31. Nose Y: Design and development strategy for the rotary blood pump. Artificial organs 22: 438-46, 1998.

32. Chan $\mathrm{CH}$, Pieper IL, Hambly R, et al: The CentriMag centrifugal blood pump as a benchmark for in vitro testing of hemocompatibility in implantable ventricular assist devices. Artificial organs 39: 93-101, 2015.

33. Bourque K, Cotter C, Dague C, et al: Design Rationale and Preclinical Evaluation of the HeartMate 3 Left Ventricular Assist System for Hemocompatibility. ASAIO journal 62: 375-83, 2016.

34. Wu WT, Yang F, Wu J, Aubry N, Massoudi M, Antaki JF: High fidelity computational simulation of thrombus formation in Thoratec HeartMate II continuous flow ventricular assist device. Scientific reports 6: 38025, 2016.

35. Wiegmann L, Thamsen B, de Zelicourt D, et al: Fluid Dynamics in the HeartMate 3: Influence of the Artificial Pulse Feature and Residual Cardiac Pulsation. Artificial organs 43: 363-376, 2019.

36. Lu Q, Hofferbert BV, Koo G, Malinauskas RA: In vitro shear stress-induced platelet activation: sensitivity of human and bovine blood. Artificial organs 37: 894903, 2013.

37. Bartoli CR, Restle DJ, Zhang DM, Acker MA, Atluri P: Pathologic von Willebrand factor degradation with a left ventricular assist device occurs via two distinct mechanisms: mechanical demolition and enzymatic cleavage. The Journal of thoracic and cardiovascular surgery 149: 281-9, 2015.

38. Kang J, Zhang DM, Restle DJ, et al: Reduced continuous-flow left ventricular assist device speed does not decrease von Willebrand factor degradation. The Journal of thoracic and cardiovascular surgery 151: 1747-1754.e1, 2016.

39. Meyer AL, Malehsa D, Budde U, Bara C, Haverich A, Strueber M: Acquired von Willebrand syndrome in patients with a centrifugal or axial continuous flow left ventricular assist device. JACC Heart failure 2: 141-5, 2014.

40. Kubicki R, Stiller B, Kroll J, et al: Acquired von Willebrand syndrome in paediatric patients during mechanical circulatory support. European journal of cardiothoracic surgery 55: 1194-1201, 2019. 
Figure 1. Image of the blood circulation loops. HVAD, HeartWare Ventricular Assist Device; Pout, outlet pressure; Pin, inlet pressure; flow meter; resistance clamp; blood reservoir.

Figure 2. Pressure and flow curves for the blood flow $(0-10 \mathrm{~L} / \mathrm{min})$ at a constant hematocrit of $30 \pm 2 \%$ bovine blood ( viscosity of $2.6 \mathrm{cP}$ ) at various pump speeds $(1900-3600$ RPM) of HVAD throughout a wide range of pressures $(0-200 \mathrm{mmHg})$ at $37 \pm 1^{\circ} \mathrm{C}$.

Figure 3. A) Hemolysis evaluation. Comparison of changes in plasma free haemoglobin $(\mathrm{pfHb})$ between adult systemic, pediatric systemic and adult pulmonary flow conditions. The degree of an increase in $p f H b$ overtime was different between the systemic and the other two conditions; the change was steeper with systemic condition than the pediatric and pulmonary conditions. ${ }^{*}$ Statistically significant difference when compared with 5 min samples. B) Calculated Normalised Index of Hemolysis $(\mathrm{NIH})$.

Figure 4. Platelet activation evaluation. Whole blood sampled every $120 \mathrm{~min}$ from the blood circulation loops and single-stained with CAPP2A antibody. There was a no increase in platelet activation at any condition.

Figure 5. A) Multimer analysis against time in four testing conditions (left). Plateletpoor plasma isolated from blood sampled every $120 \mathrm{~min}$ from the blood circulation loops (static, adult systemic, pediatric systemic and adult pulmonary conditions) and analyzed by immunoblotting. Red dotted box represents High-Molecular-Weight von Willebrand Factor (HMW vWF) multimer degradation against time B) Densitometry of the blot (right). Degradation in \% HMW vWF multimer density compare to 5 min as baseline against time. HMW VWF multimers significantly decreased at $360 \mathrm{~min}$ in all conditions except the static condition. * Statistically significant difference when compared with 5 min samples.

Table 1. Adult systemic $(n=4)$, pediatric systemic $(n=4)$ and adult pulmonary $(n=$ 4) hemodynamic parameters flow regimes of the HeartWare HVAD. 


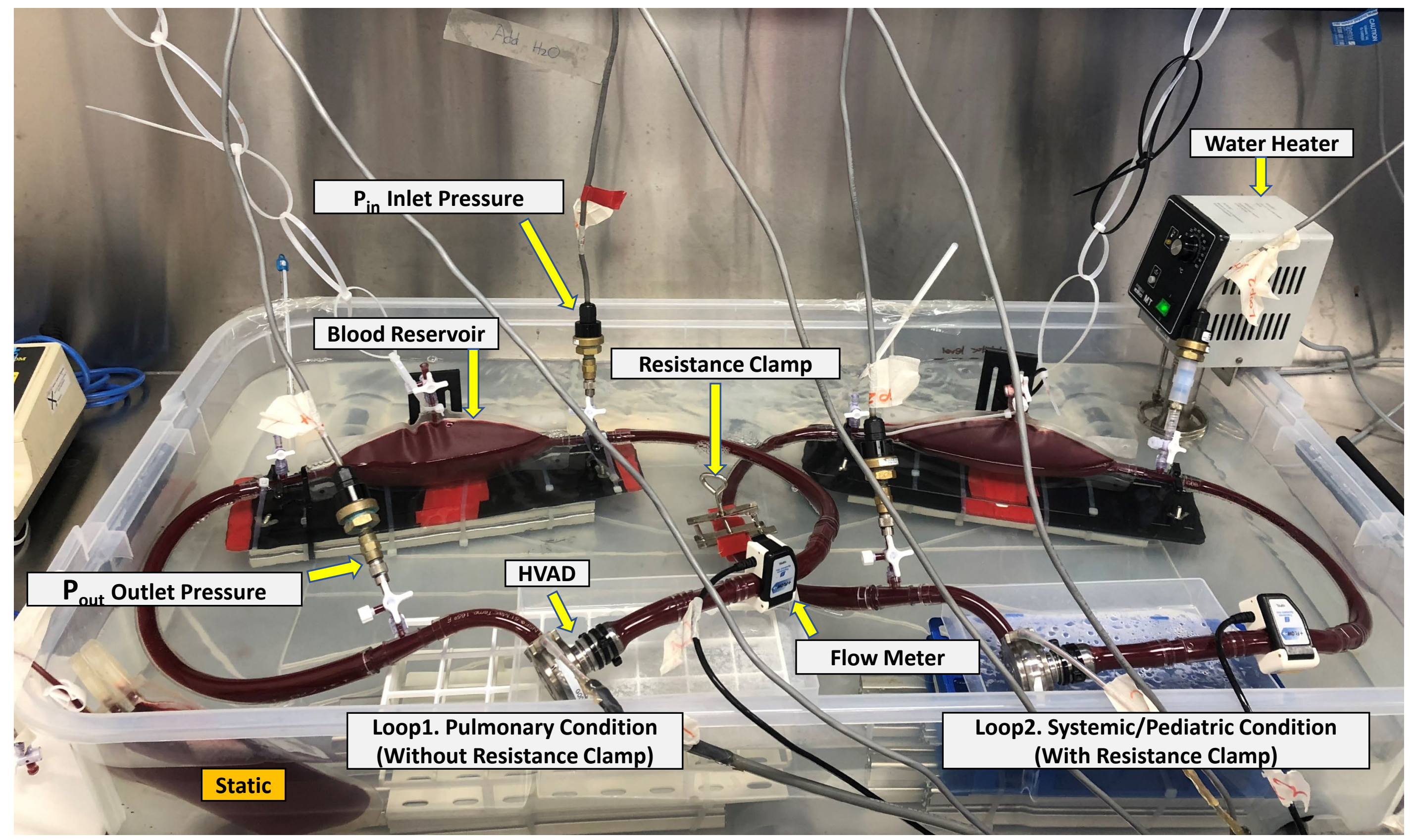

Figure 1. 


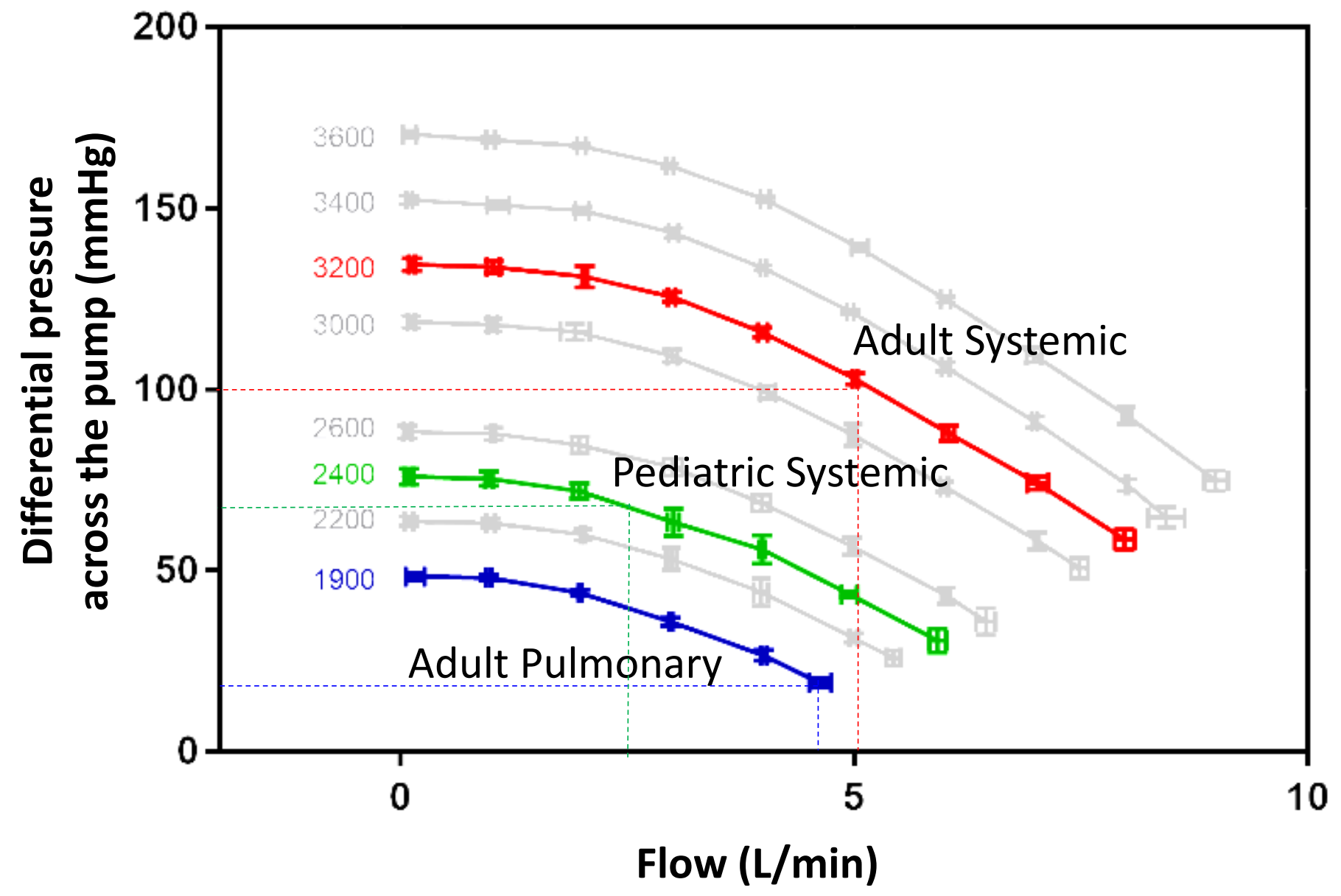

Figure 2. 
A)

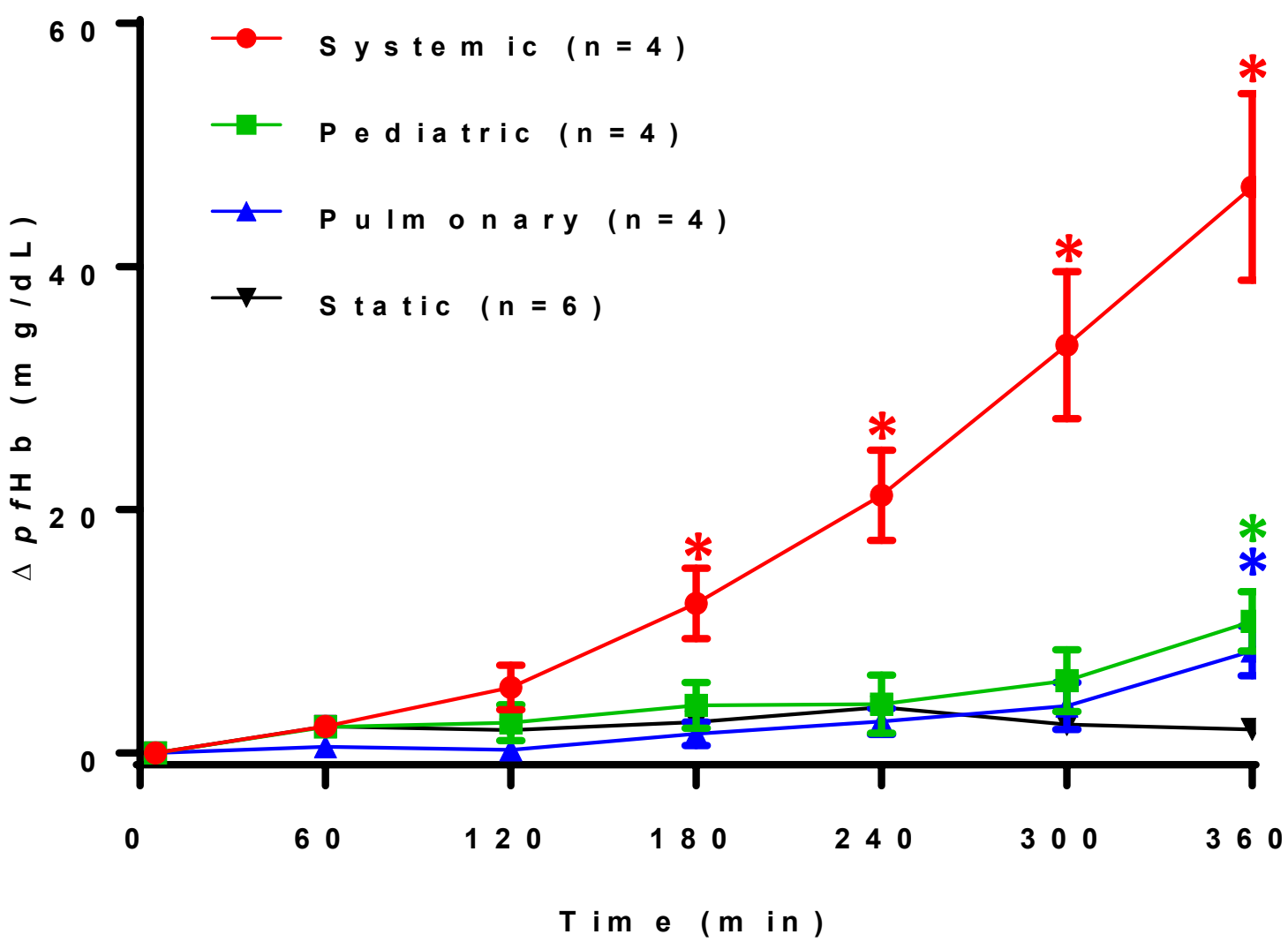

B)

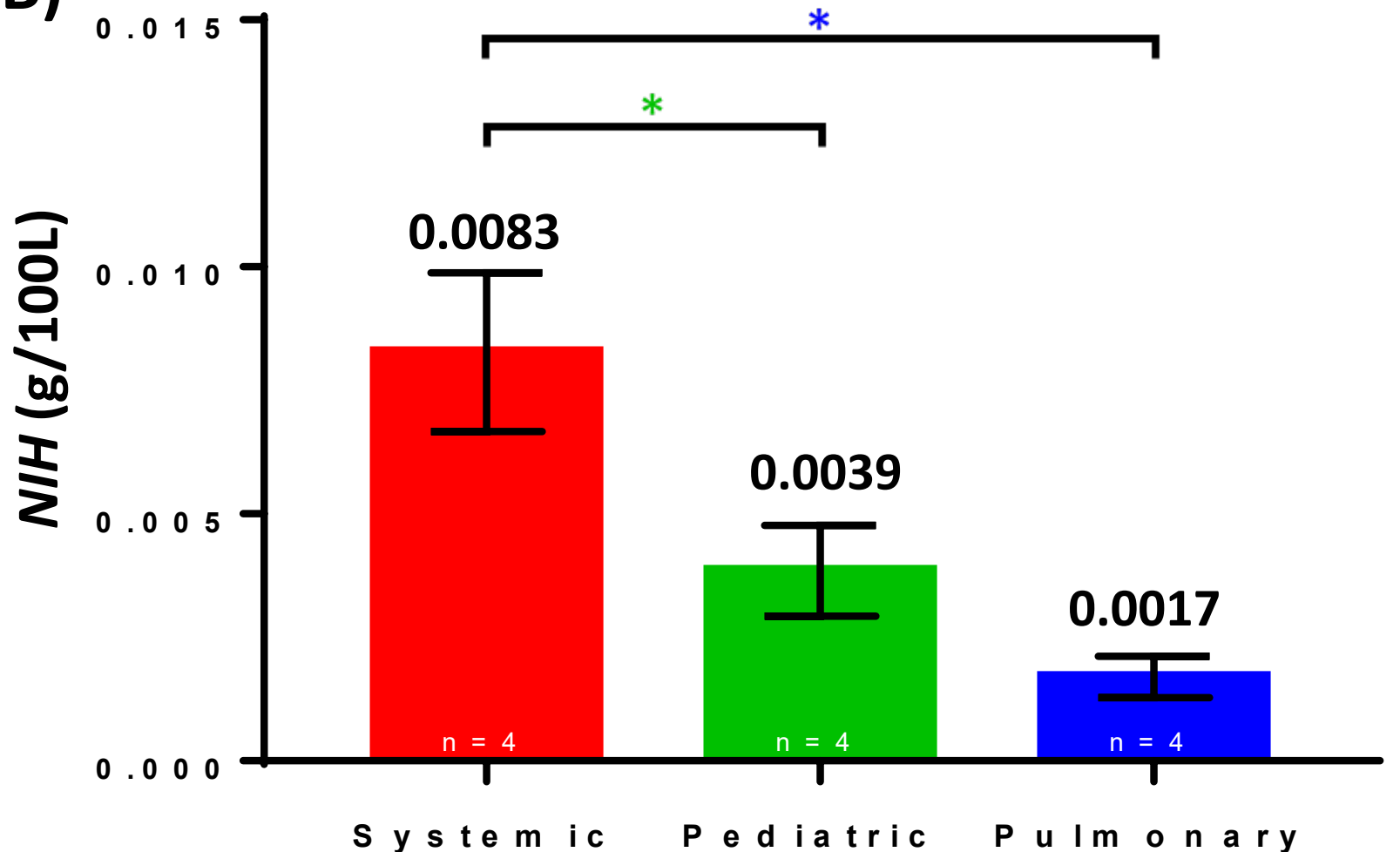

Figure 3. 


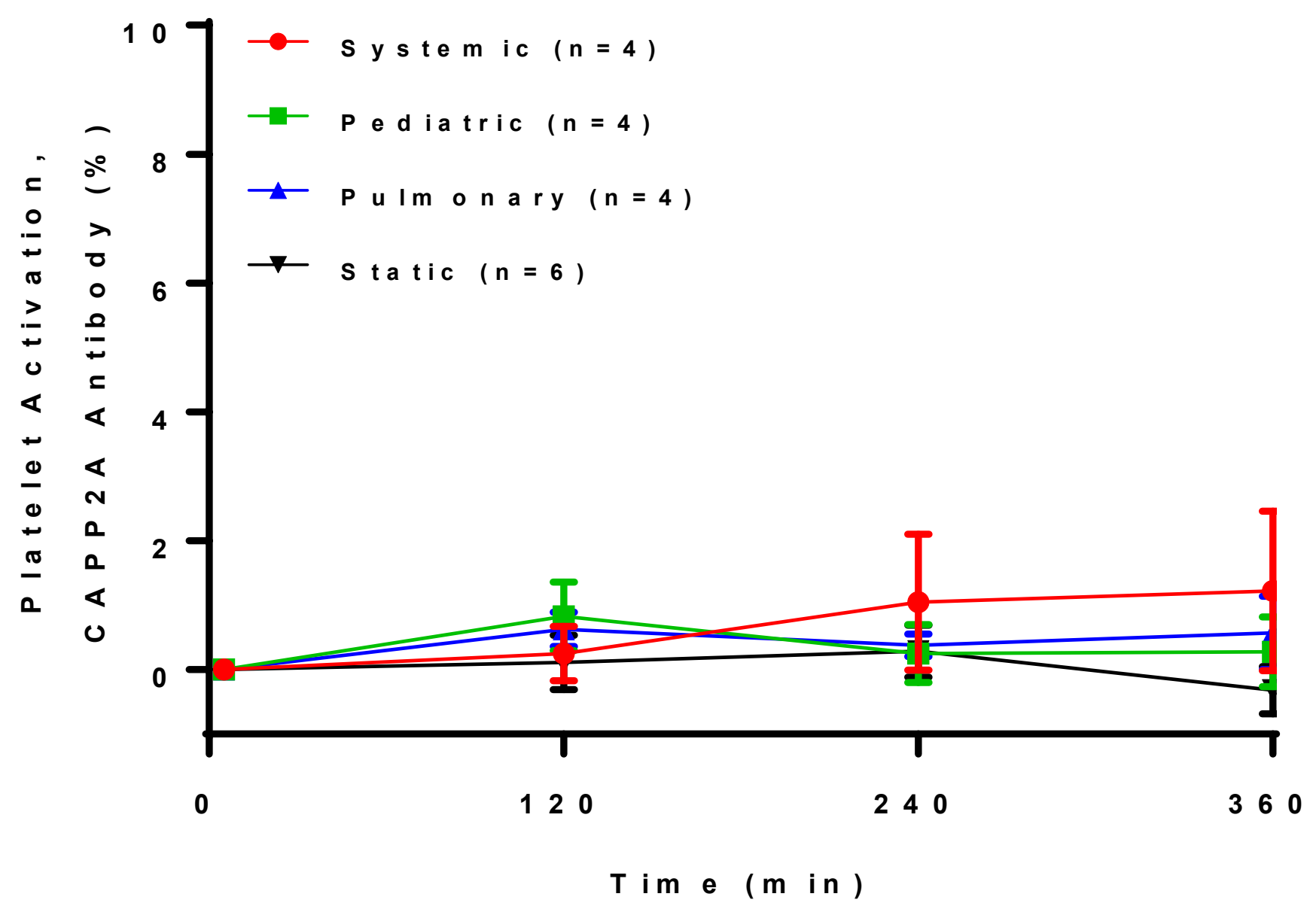

Figure 4. 
A) HMW vWF section

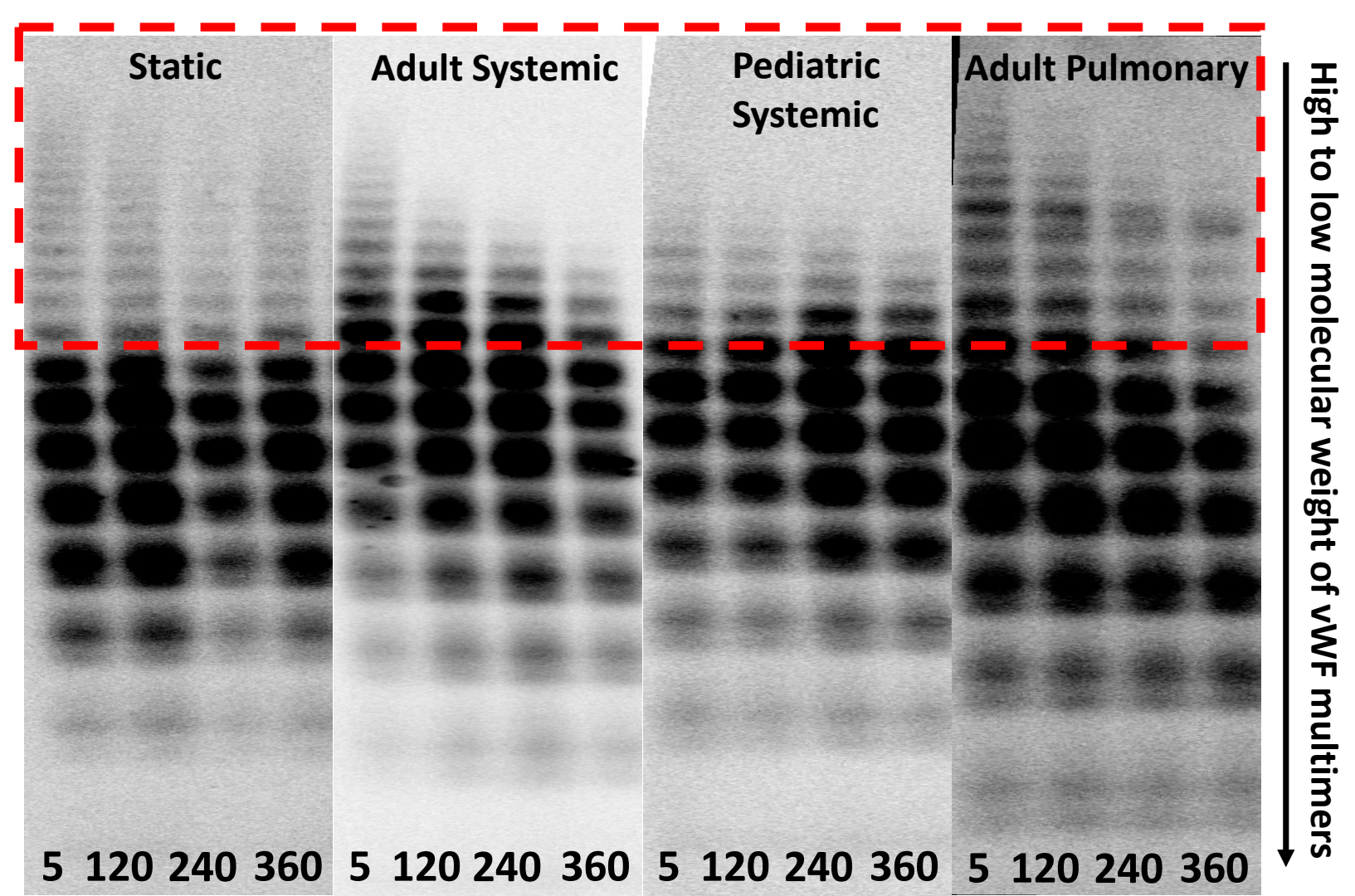

Time (min)
B)

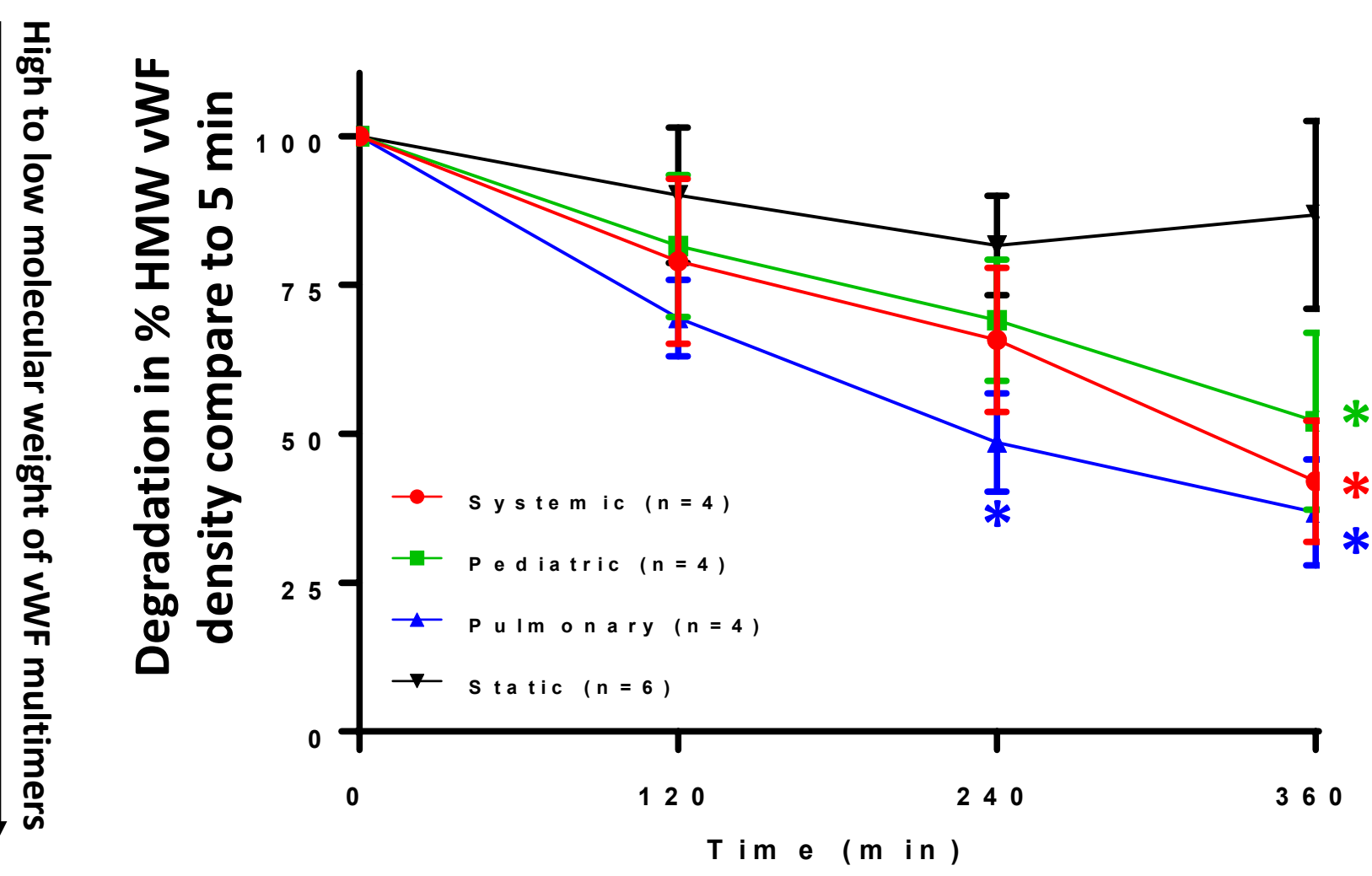

Figure 5. 